\title{
Accuracy of Ultrasound Examination of Loco-Regional Lymph Nodes in Breast Cancer Follow-Up and Its Role in the Axillary Surgical Management
}

\author{
Jacopo Nori ${ }^{1}$, Icro Meattini ${ }^{2}$, Dalmar Abdulcadir ${ }^{3 *}$, Elisabetta Giannotti ${ }^{3}$, Diego De Benedetto ${ }^{3}$, \\ Luis Sanchez ${ }^{4}$, Lorenzo Orzalesi ${ }^{4}$, Simonetta Bianchi ${ }^{5}$, Leonardo Capaccioli ${ }^{3}$, Lorenzo Livi $^{2}$ \\ ${ }^{1}$ Diagnostic Senology Unit, AOU Careggi, Florence, Italy \\ ${ }^{2}$ Radiotherapy Unit, Clinical Physiopathology Department, AOU Careggi, Florence, Italy \\ ${ }^{3}$ Department of Radiology, AOU Careggi, Florence, Italy \\ ${ }^{4}$ Breast Unit, Department of Surgery and Translational Medicine, AOU Careggi, Florence, Italy \\ ${ }^{5}$ Pathological Anatomy Unit, Department of Surgery and Translational Medicine, AOU Careggi, Florence, Italy \\ Email: *dalmar.abdulcadir@gmail.com
}

Received October 28, 2013; revised November 25, 2013; accepted December 3, 2013

Copyright (C) 2014 Jacopo Nori et al. This is an open access article distributed under the Creative Commons Attribution License, which permits unrestricted use, distribution, and reproduction in any medium, provided the original work is properly cited. In accordance of the Creative Commons Attribution License all Copyrights (C) 2014 are reserved for SCIRP and the owner of the intellectual property Jacopo Nori et al. All Copyright (C 2014 are guarded by law and by SCIRP as a guardian.

\section{ABSTRACT}

Objective: Major international guidelines have not standardized the sequence of diagnostic examinations during the follow-up of a patient with a diagnosed breast cancer. The aim of this study is to investigate the accuracy of sonography in the diagnosis of loco-regional lymphatic recurrences in comparison to the core needle biopsy results. Materials and Methods: Among 6455 patients who were followed up with clinical examination, mammography and ultrasound between January 2004 and November 2011, $125(1.93 \%)$ patients had to be investigated with a core needle biopsy of a sonographically suspicious loco-regional lymph node. Results: Among the whole series, a total of 142 ultrasound-guided core needle biopsies were performed. Follow-up for the primary tumor lasted for a median time of 6.1 years (range 1 - 27 years). Ultrasound of suspicious loco-regional lymph nodes showed a sensitivity of $\mathbf{8 9 . 5 \%}$, a specificity of $87.1 \%$ and a positive predictive value of $\mathbf{8 9 . 5 \%}$. Conclusions: In our experience, ultrasound of suspicious loco-regional lymph nodes showed good accuracy and it should be a part of the standard examinations performed during follow-up for breast cancer.

\section{KEYWORDS}

Ultrasound; Loco-Regional Lymph Nodes; Breast Cancer Follow-Up; Sentinel Node Dissection; Axillary Surgery

\section{Introduction}

The aims of the breast cancer (BC) follow-up are to find early locoregional and distant recurrences, to evaluate and treat therapies complications and to provide psychological support and information to help patients return to normal life.

A BC locoregional recurrence (LRR) is defined as the re-appearance of disease in the area of the primary treatment and/or in the regional lymph nodes: axillary, supra- and infra-clavicular, parasternal or the internal mammary chain [1].

\footnotetext{
"Corresponding author.
}

The risk of LRR is related to the clinical staging and it is considered as an independent risk factor for distant metastasis and death; an early diagnosis in patients clinically negative could have a positive impact on survival compared to symptomatic patients [2,3].

According to international guidelines, BC follow-up includes many investigations such as clinical examination, mammography and breast ultrasound (US). However, the 2011 European Society of Medical Oncology (ESMO) guidelines [4], have not established a specific protocol for the follow-up of a patient with $\mathrm{BC}$ as there is no evidence from randomized trials supporting any ideal sequence. Moreover, since sentinel lymph node dissec- 
tion (SLND) is becoming an increasingly used procedure [5], the role of US during follow-up may become even more important.

The aim of this study was to evaluate follow-up patients for previous BC, the diagnostic accuracy of US in studying loco-regional lymph nodes and its concordance to the core needle biopsy (CNB) reports.

\section{Materials and Methods}

\subsection{Patients' Series}

Between January 2004 and November 2011, at the Diagnostic Senology Unit of Careggi Florence University Hospital, 6455 patients were followed-up for a previous BC, 125 of them (1.93\%) underwent a CNB of the loco regional lymph nodes to confirm or exclude a suspect nodal recurrence diagnosed with US.

All patients underwent clinical examination, mammography and US of the breast and loco regional lymph nodes; all the exams were performed by expert radiologists in breast imaging (>10 years of experience). Our analyses comprehend only those patients who, after surgical treatment (radical or conservative surgery), needed a second lymph node biopsy during BC follow-up.

Hormonal status was defined positive in case of estrogen and/or progesterone receptors status $>10 \%$. BC was classified according to the histological type and the TNM Classification of malignant tumors [6].

\subsection{US Evaluation}

Lymph node US included axillary regions, intercostal spaces, bilateral neck, supraclavicular and subclavicular spaces. Both ipsilateral and contralateral lymph node stations were evaluated.

Sonographic examination was performed using a broadband 10 - 13 Mhz linear transducer (Technos Mylab 70 XS; Esaote; Genoa, Italy). The characteristics that were considered important to distinguish benign from malignant nodes were: size of the node, morphology, echogenicity of the hilum, focal or irregular thickening of the cortex, vascularization, proportion between the total diameter of the node and the diameter of the hilum (normal proportion when superior to $50 \%$ of the total diameter of the node). Malignant features on the US examination were: round morphology, irregular cortex, peripheral vascularization at the US sonogram, and disappearance of the hyperchogenicity of the hilum.

Considering these criteria, all nodes were graded into the five categories proposed by the European Society of Mastology (EUSOMA) [7]: U1 and U2 when the appearance of the node was either normal or generally benign; U3 when the node was suspicious but with benign features; U4 when the node was suspicious with malignant features and U5 when the node was probably ma- lignant.

When necessary (U3, U4 and U5 categories), a USguided CNB of the node was performed. All biopsies were performed under local anesthesia with a semi automated breast gun (Precisa 14-G; Hospital Service; Rome, Italy) with a 14-gauge, 15-centimeter long needle; on average, 4 core samples (range 3 - 8) per lesion were obtained.

US sensitivity in suspicious node was calculated as the rate of true positive U5 evaluations among positive biopsied cases and specificity as the rate of true negative U3 evaluations among negative biopsied cases. Positive predictive value was calculated as the true positive U5 evaluations among all the U5 evaluations.

\section{Results}

All 125 patients were women with a median age at the diagnosis of $\mathrm{BC}$ of 53.5 years (range 18 - 85 years). Among characteristics at primary BC diagnosis, 15 patients $(12 \%)$ were stage IIA, 31 patients $(24.8 \%)$ were stage IIB and 79 (63.2\%) were stage IIIA; concerning nodal status, 15 patients (12\%) were node-negative. Hormonal status was positive in 102 patients (81.6\%); HER2 status was available in 95 cases and was positive in 13 patients (13.7\%).

In all cases the primary BC was surgically treated: 47 patients (37.6\%) underwent mastectomy and axillary lymph node dissection (ALND), 78 patients (62.4\%) underwent a quadrantectomy followed by either ALND (47 patients; 37.6\%) or SLND (31 patients; 24.8\%). Followup for the primary tumor lasted for a median period of time of 6.1 years (range 1 - 27 years).

Main recurrences features stratified by definitive nodal status are summarized in Table 1; metastatic lymph node sites in relation to the type of surgery are reported in Table 2.

Among the whole series, 142 US-guided CNB were performed: sonographic appearances of sampled lymph nodes are listed in Table 3. Major sonographic findings of vascular and morphological alterations in malignant lesions diagnosed by US examination are showed in Figures 1 and 2, respectively.

Concerning concordance between US appearance and CNB results, US examination in case of suspicious node, showed a sensitivity of $89.5 \%$ (true positive among positive biopsied cases) and a specificity of $87.1 \%$ (true negative among negative biopsied cases), with a positive predictive value of $89.5 \%$ (true positive among positive US cases).

\section{Discussion}

\subsection{Indications for US Examination: Morphologic Criteria}

Our study considered the use of the US examination of 
Table 1. Patients and core-needle biopsies characteristics stratified by definitive histological result.

\begin{tabular}{cccc}
\hline & $\begin{array}{c}\text { Positive nodal status after US-guided } \\
\text { CNB, n (\%) }\end{array}$ & $\begin{array}{c}\text { Negative nodal status after US-guided } \\
\text { CNB, } \mathrm{n}(\%)\end{array}$ & Total, $\mathrm{n}(\%)$ \\
\hline Surgery & & & \\
Mastectomy + ALND & $25(20)$ & $22(17.6)$ & $47(37.6)$ \\
BCS + ALND & $13(10.4)$ & $34(27.2)$ & $47(37.6)$ \\
BCS + SLND & $17(13.6)$ & $14(11.2)$ & $31(24.8)$ \\
Total & $55(44)$ & $70(56)$ & $125(100)$ \\
Median age, years (range) & $58.5(29-85)$ & $60.7(19-85)$ & \\
CNB nodal region & & & $107(75.3)$ \\
Axilla & $33(23.2)$ & $74(52.1)$ & $18(12.7)$ \\
Sovraclavear & $13(9.2)$ & $5(3.5)$ & $5(3.5)$ \\
Subclavear & $4(2.8)$ & $1(0.7)$ & $5(3.5)$ \\
Laterocervical & $5(3.5)$ & $5(3.5)$ & $7(5)$ \\
Internal mammary & $2(1.5)$ & $85(59.8)$ & $142(100)$ \\
Total & $57(40.2)$ & & \\
\hline
\end{tabular}

Abbreviations: CNB, core-needle biopsy; BCS, breast conserving surgery; ALND, axillary lymph node dissection; SLND, sentinel lymph node dissection; US, ultrasound.

Table 2. Metastatic lymph node regions stratified by primary surgical approach.

\begin{tabular}{|c|c|c|c|c|c|c|}
\hline & \multicolumn{3}{|c|}{ Positive Nodal status after US-guided CNB, n (\%) } & \multicolumn{3}{|c|}{ Negative Nodal status after US-guided CNB, n (\%) } \\
\hline Surgical approach/Nodal region & Mastectomy + ALND & BCS + ALND & BCS + SLND & Mastectomy + ALND & BCS + ALND & BCS + SLND \\
\hline Ipsilateral axilla & $13(9.2)$ & $4(2.8)$ & $11(7.7)$ & $19(13.4)$ & $33(23.3)$ & $15(10.6)$ \\
\hline Contralateral axilla & $3(2.1)$ & $1(0.7)$ & $1(0.7)$ & $2(1.5)$ & $2(1.5)$ & $3(2.1)$ \\
\hline Sovraclavear & $6(4.1)$ & $5(3.5)$ & $2(1.5)$ & $1(0.7)$ & $4(2.8)$ & 0 \\
\hline Mammary & $1(0.7)$ & 0 & $1(0.7)$ & $1(0.7)$ & $4(2.8)$ & 0 \\
\hline Ipsilateral laterocervical & $1(0.7)$ & $3(2.1)$ & 0 & 0 & 0 & 0 \\
\hline Contralateral laterocervical & $1(0.7)$ & 0 & 0 & 0 & 0 & 0 \\
\hline Subclavear & 0 & $2(1.5)$ & $2(1.5)$ & $1(0.7)$ & 0 & 0 \\
\hline Total & & $57(40.2)$ & & & 85 (59.8) & \\
\hline
\end{tabular}

Abbreviations: CNB, core-needle biopsy; BCS, breast conserving surgery; ALND, axillary lymph node dissection; SLND, sentinel lymph node dissection; US, ultrasound.

Table 3. Sonographic appearance of 142 sampled lymph nodes.

\begin{tabular}{cccc}
\hline Sonographic appearance & Positive nodal status after US-guided CNB, $\mathrm{n}(\%)$ & Negative nodal status after US-guided CNB, $\mathrm{n}(\%)$ & Total \\
\hline U3 & $3(2.1)$ & $74(52.1)$ & $77(54.2)$ \\
U4 & $3(2.1)$ & $5(3.5)$ & $8(5.6)$ \\
U5 & $51(36)$ & $6(4.2)$ & $57(40.2)$ \\
Total & $57(40.2)$ & $85(59.8)$ & $142(100.0)$ \\
\hline
\end{tabular}

Abbreviations: CNB, core-needle biopsy; US, ultrasound.

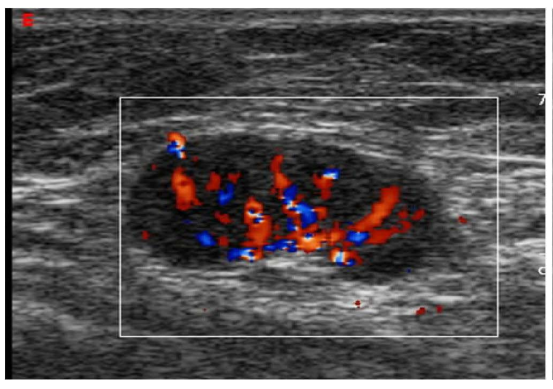

(a)

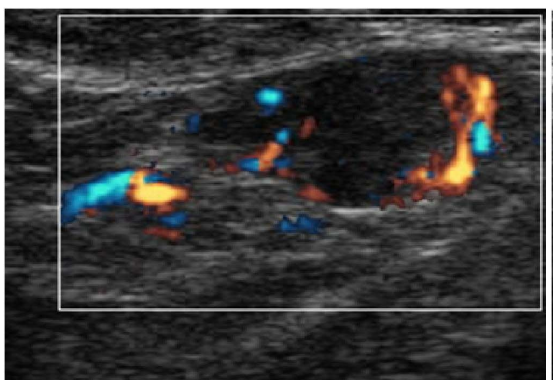

(b)

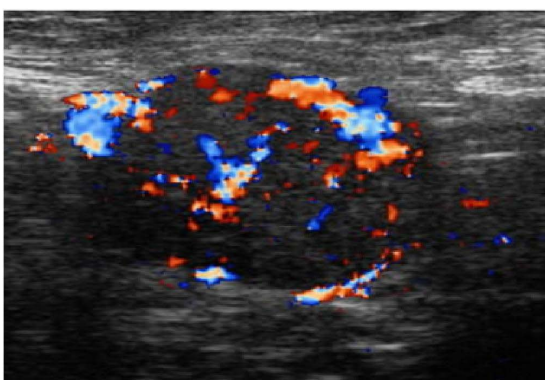

(c)

Figure 1. Major sonographic findings of vascular alterations in malignant lesions diagnosed by US examination: a case of aberrant vessels (a), focal absence (b), subcapsular vessels (c). 


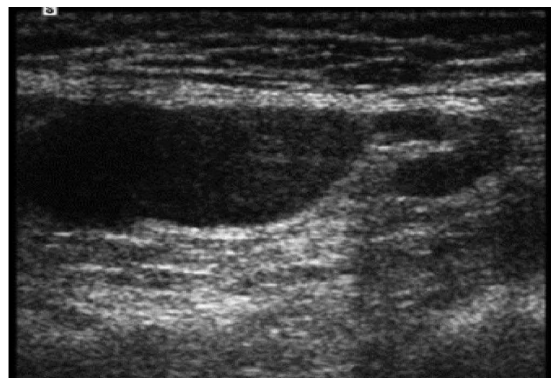

(a)

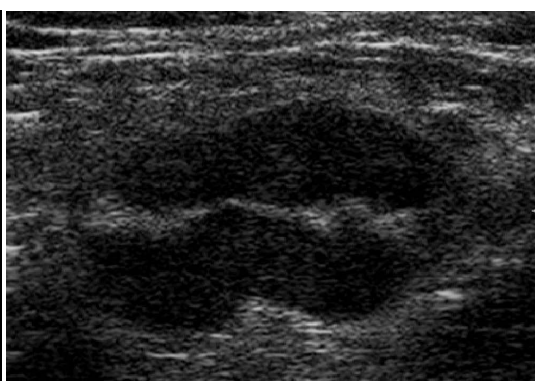

(b)

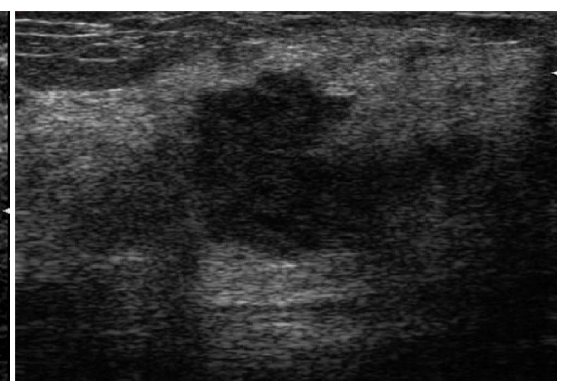

(c)

Figure 2. Major sonographic findings of morphological alterations in malignant lesions diagnosed by US examination: a case of asymmetric thickening of the cortex (a), concentric compression of the hilum (b), irregular capsular margins (c).

the axilla with an eventual CNB in the routine follow-up of patients treated for BC, following the EUSOMA guidelines [7].

The US examination of regional lymph nodes assessed the possible presence of metastases through the following criteria: shape, size, echogenicity, morphology of nodal cortex and hilum, hilar vascularity [8-11].

The presence of a single abnormality may not be sufficient to suspect if the lymph node is malignant. Metastatic lymph nodes appear globular in shape or round with a hypoechoic structure and lack the corticomedullary differentiation. Concerning nodal echogenicity and morphology, metastatic lymph nodes lose the hilar hyperechogenicity because the malignant cells tend to invade the cortical and subcortical region that leads to a deformity of the hilum.

In addition, nodal size increases US accuracy evaluation. Alvarez et al. [12] evaluated the sensitivity and specificity of morphological criteria versus the size of lymph nodes. Considering lymph node morphology, US sensitivity ranged from $48.8 \%$ to $87.1 \%$ and specificity ranged from $55.6 \%$ to $97.3 \%$. Size alone had a sensitivity varying between $26.4 \%$ and $75.9 \%$ whereas specificity ranged between $88.1 \%$ and $98.1 \%$.

\subsection{Accuracy of US Examination in BC Follow-Up}

There are only a few studies investigating the diagnostic accuracy of US in BC follow-up.

Shin et al. [13] evaluated the effectiveness of the US in the diagnosis of LRR in clinically asymptomatic patients with a negative mammogram. The Authors enrolled 1968 patients, showing an US sensitivity and specificity of $70.6 \%$ and $98.3 \%$, respectively.

We have already published concerning the importance of US in symptomatic patients (with palpable lymph nodes) undergoing SLND [14]. Since SLND is becoming an increasingly used procedure, it is advisable a much more extensive use of US to detect an axillary recurrence during the asymptomatic phase as many studies reported a better control of the disease, if diagnosed at an early stage.

Many Authors suggest the use of US in the follow-up also in asymptomatic patients. Moon et al. [15] enrolled 1817 patients who were investigated with US in the axilla and in the supraclavicular areas bilaterally; they reported 54 cases of suspected lymph node LRR, but the diagnosis was confirmed in 39 cases (10 of them were contralateral). The reported sensitivity and specificity of the US examination was respectively $78 \%$ and $99.4 \%$, while the accuracy was $99.2 \%$.

Kim et al. [3] studied the LRR with US in 874 asymptomatic patients treated with mastectomy for BC. During a mean follow-up of 37 months the Authors found 22 suspicious lesions that were histologically confirmed in 6 cases. The reported values of sensitivity, specificity and accuracy were $78.9 \%, 95.1 \%$ and $94.7 \%$, respectively.

Lee et al. [16] assessed the value of US screening in the detection of non-palpable LRR following mastectomy in 1180 consecutive cases. The sensitivity and specificity were $90.9 \%$ and $98.0 \%$, respectively. A biopsy positive predictive value of $52.6 \%$ was observed. The Authors concluded that although LRR infrequently occurs after mastectomy for BC, routine US follow-up could be advocated for early detection of recurrent BC.

Concerning concordance between US appearance and CNB results, in our experience US examination showed a sensitivity of $89.5 \%$ and a specificity of $87.1 \%$, with a positive predictive value of $89.5 \%$.

We recorded 11 false positive U4-5 cases (7.7\%), probably due to the interoperator variability in assigning the sonographic score and the lack of standardized criteria that may favor an overestimated rate of suspicious (U4) or malignant (U5) lymph nodes.

The role of US-guided CNB in the staging and follow-up of BC is well-known, with an accuracy of around $100 \%[17,18]$.

In our study the combined use of CNB helped to confirm or rule out the US score, and it allowed avoiding expensive examinations, such as PET scans. No sampling errors were found, each CNB was suitable for the final 
diagnosis of the suspicious lymph nodes.

Conversely, negative known side effects of US examination are the additional costs, the risk of a higher overall biopsy rate and an elevated benign biopsies rate; so the advantages and disadvantages of additional breast US should be discussed for every follow-up patient individually [19].

However we can detect a significantly higher number of previously occult malignancies. Moreover, it has to be considered that in our department, patients undergo routinely breast US during the follow-up. Therefore, for an experienced radiologist, at the end of the breast US examination it takes a few seconds to scan the loco-regional drainages as well, and the additional cost is zero [14].

\subsection{Update of Surgical Management of Axillary Region in Adjuvant Breast Cancer Treatment}

Total ALND has been for a long time the gold standard of surgical axillary therapy for BC. Since the diagnosis is nowadays made in an early stage, the possibility of a lymph node involvement is lower than the past [20]. The technique of SLND is now widely used and could largely replace ALND, especially when the sentinel node is negative [21]. On the other hand there is still a debate in case this is positive (minimal axillary involvement, micrometastases or isolated tumor cells) [5,22]. According to some relevant published papers [20,23], there is evidence that occult axillary metastasis may never become clinically evident.

In our study, LRR occurred in 38 of 94 (40.4\%) patients treated with surgery and ALND and in 17 of 31 (54.8\%) patients treated with surgery and SLND.

Van der Ploeg et al. [24] published a meta-analysis evaluating 48 papers concerning the incidence of axillary recurrence in BC patients treated with SLND. Among 14 959 patients, only 67 (0.3\%) developed a LRR, after an average follow-up time of 34 months. The Authors reports also that the LRR rate is influenced by the differences in the lymphatic mapping technique.

Takei et al. [25] studied the pattern and the risk factors associated to the LRR. They enrolled and followed-up 1670 patients for a median time of 34 months (2 - 83 months) and found an axillary recurrence rate of $0.5 \%$. In addition, the authors reported that in those patients who were treated with ALND, despite negative sentinel node pathology, recurrences were prevented in only the $0.4 \%$ of the patients.

According to these results, Veronesi et al. [26] reported that among 3548 patients treated with SLND, only 31 patients $(0.9 \%)$ developed an axillary recurrence after 86 months from the surgery.

Bernardi et al. [27] evaluated the incidence of axillary recurrence in 1050 patients treated in a single Italian center, subjected to SLND. LRR were found to be $1 \%$ after a mean follow-up of 54 months and only $1.7 \%$ after a median time of 6 years.

Concerning the ideal axillary surgical approach, several trials are addressing the problem (IBCSG 23-01, ASCOG Z0011, EORTC AMAROS) [23,28,29]. Z0011 has published interim results, finding, after a median follow-up of 6.3 years, no differences in LRR or regional recurrence between patients, with a positive $\mathrm{SN}$, who received ALND versus no further axillary treatment. If axillary dissection is not always necessary in women with a positive axilla, it seems important to be able to reliably identify the patients at high risk of developing overt axillary disease who should receive elective ALND.

Concerning IBCSG 23-01 trial, the Authors recently published first results [30], with 5-year disease-free survival of $87.8 \%$ in the group without ALND and $84.4 \%$ in the group with ALND (log-rank $\mathrm{p}=0.16$; HR for no axillary dissection vs axillary dissection of 0.78 , noninferiority $p=0.0042$ ). The authors concluded that ALND could be avoided in patients with early breast cancer and limited sentinel-node involvement, thus eliminating complications of axillary surgery with no adverse effect on survival.

Ancillary analyses of the AMAROS trial, still in follow-up, may be able to add more significant data.

\section{Conclusion}

In our experience, US in suspicious nodal recurrences showed good accuracy with a sensitivity of $89.5 \%$ and a specificity of $87.1 \%$. Since SLND is becoming an increasingly used procedure, a much more extensive use of US to detect an occult nodal LRR during BC follow-up is advisable.

\section{REFERENCES}

[1] H. Rauschecker, M. Clarke, W. Gatzemeier and A. Recht, "Systemic Therapy for Treating Locoregional Recurrence in Women with Breast Cancer," Cochrane Database of Systematic Reviews, 2001, CD002195.

[2] F. Aydogan, V. Ozben, D. Atasoy, M. H. Yilmaz, M. Halaç and V. Celik, "Excision of Axillary Lymph Node Recurrences in Breast Cancer Patients with Axillary ROLL (A-ROLL),” Journal of Surgical Oncology, Vol. 101, No. 2, 2010, pp. 141-144.

[3] H. J. Kim, J. Y. Kwak, J. W. Choi, et al., "Impact of US Surveillance on Detection of Clinically Occult Locoregional Recurrence after Mastectomy for Breast Cancer," Annals of Surgical Oncology, Vol. 17, No. 10, 2010, pp. 2670-2676. http://dx.doi.org/10.1245/s10434-010-1087-z

[4] S. Aebi, T. Davidson, G. Gruber, F. Cardoso and ESMO Guidelines Working Group, "Primary Breast Cancer: ESMO Clinical Practice Guidelines for Diagnosis, Treat- 
ment and Follow-Up,” Annals of Oncology, Vol. 22, Suppl. 6, 2011, pp. S12-S24.

http://dx.doi.org/10.1093/annonc/mdr371

[5] H. Torrenga, H. Fabry, J. R. van der Sijp, P. J. van Diest, R. Pijpers and S. Meijer, "Omitting Axillary Lymph Node Dissection in Sentinel Node Negative Breast Cancer Patients Is Safe: A Long Term Follow-Up Analysis,” Journal of Surgical Oncology, Vol. 88, No. 1, 2004, pp. 4-7. http://dx.doi.org/10.1002/jso.20101

[6] L. Sobin, M. Gospodarowicz and C. Wittekind, Eds., "UICC TNM Classification of Malignant Tumors," 7th Edition, John Wiley \& Sons, Inc., Hoboken, 2009.

[7] N. M. Perry and EUSOMA Working Party, "Quality Assurance in the Diagnosis of Breast Disease. EUSOMA Working Party," European Journal of Cancer, Vol. 37, No. 2, 2001, pp. 159-172. http://dx.doi.org/10.1016/S0959-8049(00)00337-3

[8] H. Ojeda-Fournier and J. Q. Nguyen, "Ultrasound Evaluation of Regional Breast Lymph Node," Seminars in Roentgenology, Vol. 46, No. 1, 2011, pp. 51-59. http://dx.doi.org/10.1053/j.ro.2010.06.007

[9] F. Sakai, K. Kiyono, S. Sone, et al., "Ultrasonic Evaluation of Cervical Metastatic Lymphadenopathy,” Journal of Ultrasound in Medicine, Vol. 7, No. 6, 1988, pp. 305310.

[10] A. Luparia, P. Campanino, R. Cotti, et al., "Role of Axillary Ultrasound in the Preoperative Diagnosis of Lymph Node Metastases in Patients Affected by Breast Carcinoma,” Radiologia Medica, Vol. 115, No. 2, 2010, pp. 225-237. http://dx.doi.org/10.1007/s11547-009-0465-8

[11] J. Nori, E. Vanzi, M. Bazzocchi, et al., "Role of Axillary Ultrasound Examination in the Selection of Breast Cancer Patients for Sentinel Node Biopsy,” American Journal of Surgery, Vol. 193, No. 6, 2007, pp. 16-20. http://dx.doi.org/10.1016/j.amjsurg.2006.02.021

[12] S. Alvarez, E. Añorbe, P. Alcorta, et al., "Role of Sonography in the Diagnosis of Axillary Lymph Node Metastases in Breast Cancer: A Systematic Review," American Journal of Roentgenology, Vol. 186, No. 5, 2006, pp. 13421348. http://dx.doi.org/10.2214/AJR.05.0936

[13] J. H. Shin, B. K. Han, Y. H. Choe, et al., "Ultrasonographic Detection of Occult Cancer in Patients after Surgical Therapy for Breast Cancer,” Journal of Ultrasound in Medicine, Vol. 24, No. 5, 2005, pp. 643-649.

[14] T. Susini, J. Nori, E. Vanzi, et al., “Axillary Ultrasound Scanning in the Follow-Up of Breast Cancer Patients Undergoing Sentinel Node Biopsy,” Breast, Vol. 16, No. 2, 2007, pp. 190-196. http://dx.doi.org/10.1016/j.breast.2006.10.004

[15] H. J. Moon, M. J. Kim, E. K. Kim, et al., "US Surveillance of Regional Lymph Node Recurrence after Breast Cancer Surgery,” Radiology, Vol. 252, No. 3, 2009, pp. 673-681. http://dx.doi.org/10.1148/radiol.2523081977

[16] J. H. Lee, E. K. Kim, J. Y. Oh, et al., "US Screening for Detection of Nonpalpable Locoregional Recurrence after Mastectomy," European Journal of Radiology, Vol. 82, No. 3, 2013, pp. 485-489.

http://dx.doi.org/10.1016/j.ejrad.2012.10.007
[17] H. Abe, R. A. Schmidt, K. Kulkarni, C. A. Sennett, J. S. Mueller and G. M. Newstead, "Axillary Lymph Nodes Suspicious for Breast Cancer Metastasis: Sampling with US-Guided 14-Gauge Core-Needle Biopsy-Clinical Experience in 100 Patients,” Radiology, Vol. 250, No. 1, 2009, pp. 41-49. http://dx.doi.org/10.1148/radiol.2493071483

[18] H. Abe, R. A. Schmidt, C. A. Sennett, A. Shimauchi and G. M. Newstead, "US-Guided Core Needle Biopsy of Axillary Lymph Nodes in Patients with Breast Cancer: Why and How to Do It,” Radiographics, Vol. 27, Suppl. 1, 2007, pp. S91-S99. http://dx.doi.org/10.1148/rg.27si075502

[19] S. L. Koelliker, M. A. Chung, M. Mainiero, et al., “Axillary Lymph Nodes: US-Guided Fine-Needle Aspiration for Initial Staging of Breast Cancer-Correlation with Primary Tumor Size,” Radiology, Vol. 246, No. 1, 2008, pp. 81-89. http://dx.doi.org/10.1148/radiol.2463061463

[20] S. Wojcinski, A. Farrokh, U. Hille, et al., "Optimizing Breast Cancer Follow-Up: Diagnostic Value and Costs of Additional Routine Breast Ultrasound," Ultrasound in Medicine and Biology, Vol. 37, No. 2, 2011, pp. 198-206. http://dx.doi.org/10.1016/j.ultrasmedbio.2010.10.024

[21] U. Veronesi, R. Orecchia, S. Zurrida, et al., “Avoiding Axillary Dissection in Breast Cancer Surgery: A Randomized Trial to Assess the Role of Axillary Radiotherapy,” Annals of Oncology, Vol. 16, No. 3, 2005, pp. 383-388. http://dx.doi.org/10.1093/annonc/mdi089

[22] I. M. Van der Ploeg, B. B. Kroon, N. Antonini, et al., "Axillary and Extra-Axillary Lymph Node Recurrences after a Tumor-Negative Sentinel Node Biopsy for Breast Cancer Using Intralesional Tracer Administration,” Annals of Surgical Oncology, Vol. 15, No. 4, 2008, pp. 1025-1031. http://dx.doi.org/10.1245/s10434-007-9760-6

[23] A. E. Giuliano, L. McCall, P. Beitsch, et al., "Locoregional Recurrence after Sentinel Lymph Node Dissection with or without Axillary Dissection in Patients with Sentinel Lymph Node Metastases: The American College of Surgeons Oncology Group Z0011 Randomized Trial,” Annals of Surgery, Vol. 252, No. 3, 2010, pp. 426-432.

[24] I. M. Van der Ploeg, O. E. Nieweg, M. C. van Rijk, et al., "Axillary Recurrence after a Tumour-Negative Sentinel Node Biopsy in Breast Cancer Patients: A Systematic Review and Meta-Analysis of the Literature," European Journal of Surgical Oncology, Vol. 34, No. 12, 2008, pp. 1277-1284. http://dx.doi.org/10.1016/j.ejso.2008.01.034

[25] H. Takei, K. Suemasu, M. Kurosumi, et al., "Recurrence after Sentinel Lymph Node Biopsy with or without Axillary Lymph Node Dissection in Patients with Breast Cancer,” Breast Cancer, Vol. 14, No. 1, 2007, pp. 16-24. http://dx.doi.org/10.2325/jbcs.14.16

[26] U. Veronesi, V. Galimberti, G. Paganelli, et al., “Axillary Metastases in Breast Cancer Patients with Negative Sentinel Nodes: A Follow-Up of 3548 Cases,” European Journal of Cancer, Vol. 45, No. 8, 2009, pp. 1381-1388. http://dx.doi.org/10.1016/j.ejca.2008.11.041

[27] S. Bernardi, S. Bertozzi, A. P. Londero, et al., "Nine Years of Experience with the Sentinel Lymph Node Biopsy in a Single Italian Center: A Retrospective Analysis of 1050 Cases,” World Journal of Surgery, Vol. 36, No. 4, 
2012, pp. 714-722.

http://dx.doi.org/10.1007/s00268-011-1420-0

[28] A. E. Giuliano, K. K. Hunt, K. V. Ballman, et al., “Axillary Dissection vs No Axillary Dissection in Women with Invasive Breast Cancer and Sentinel Node Metastasis: A Randomized Clinical Trial,” JAMA, Vol. 305, No. 6, 2011, pp. 569-575.

http://dx.doi.org/10.1001/jama.2011.90

[29] V. Galimberti, E. Botteri, C. Chifu, et al., "Can We Avoid Axillary Dissection in the Micrometastatic Senti- nel Node in Breast Cancer?” Breast Cancer Research and Treatment, Vol. 131, No. 3, 2012, pp. 819-825. http://dx.doi.org/10.1007/s10549-011-1486-2

[30] V. Galimberti, B. F. Cole, S. Zurrida, et al., “Axillary Dissection versus No Axillary Dissection in Patients with Sentinel-Node Micrometastases (IBCSG 23-01): A Phase 3 Randomised Controlled Trial," Lancet Oncology, Vol. 14, No. 4, 2013, pp. 297-305. http://dx.doi.org/10.1016/S1470-2045(13)70035-4 